\title{
Transfixant Injuries of the Mediastinum: Always A Challenge
}

\author{
Wysterlânyo Kayo Pereira Barros, Isadora Priscila de Oliveira Sizenando, Tâmara Azevedo de \\ Medeiros, Thomas Di Nardi Medeiros, Wagner Gomes Da Nóbrega Silva, Amália Cinthia Meneses \\ Rêgo, and Irami Araújo-Filho
}

\begin{abstract}
Transfixant injuries of the mediastinum are those in which the aggressor object travels through the midline of the thorax, where the mediastinum is located, being associated with high morbidity and mortality. This review of the literature addressed several aspects of the theme, including diagnosis and treatment. A search was carried out in Pubmed, Scopus and Scielo databases, using indexed descriptors and selection of articles by peers. Mortality rates from $20 \%$ to $87 \%$ among the victims of this injury modality were observed, varying due to the proportion of unstable patients. In patients undergoing thoracotomy resuscitation, mortality rates between $85-100 \%$. The treatment consisted of maintaining the patient's hemodynamic status, so that patients with imminent death in the emergency service should undergo resuscitation thoracotomy, unstable patients should undergo emergency thoracotomy After a brief clinical/diagnostic evaluation, while stable patients benefit from further investigation and in up to $60 \%$ of the cases, do not require a surgical approach. It was concluded, therefore, that such lesions are associated with high morbidity and mortality, and their approach differs according to the patient's hemodynamic profile in the emergency room. Emergency services should be prepared to meet this new patient profile, which arrives at prompt care with severe injuries and increasingly critical clinical conditions.
\end{abstract}

Index Terms-Thorax, Penetrating Wounds, Injury, Mediastinum, Thoracotomy, Hospital Service Emergencies.

\section{INTRODUCTION}

The term mediastinum (from Latin: which is in the middle) refers to the anatomical space located between the lungs, sternal and spine. It is didactically divided in upper and lower by the transverse plane of thorax, being the last compartment subdivided in anterior (in front of the heart), medium (including pericardium) and posterior (between the pericardium and the spine) [1]-[3].

In the upper mediastinum, we find the thymus, the trachea to its bifurcation, the esophagus, the large vessels of the

Published on July 15, 2019.

W. K. P. Barros, I. P de O. Sizenando, T. A de Medeiros, T. Di N Medeiros, W. G. Da N. Silva are Medical Students of the Federal University of the State of Rio Grande do Norte - UFRN. Multicampi School of Medical Sciences of Rio Grande do Norte - Brazil.

A. C. M. Rêgo is with Postgraduate Program in Biotechnology at Potiguar University / UnP - Laureate International Universities; Pharmaceutical Biochemistry and Professor; Ph.D in Health Sciences/ Natal-RN, Brazil.

I. Araújo-Filho is with Postgraduate Program in Biotechnology at Potiguar University/ UnP - Laureate International Universities. Full Professor, Department of Surgery, Federal University of Rio Grande do Norte. Full Professor, Department of Surgery, Potiguar University. Ph.D in Health Science/ Natal-RN, Brazil.

Corresponding author: irami.filho@uol.com.br heart, among other structures [4]. The lower-anterior mediastinum contains only the retrosternal lymphatic drainage of the breasts, while the lower-middle mediastinum is filled by the pericardium, which includes the heart and the ascending part of the aorta [5].

The inferior-posterior mediastinum is home to the esophagus, descending part of the aorta, the system of the azygos vein, the vagus nerve, the sympathetic trunk and its branches, thoracic duct, blood vessels, and intercostal nerves [6], [7].

Transfixant mediastinum injuries can be defined as those in which the aggressor object crosses from one side to the other midline of the thorax. This type of damage is caused by firearms in most cases, although it may also be produced by white weapons or other forms of penetrating agents [1][4]. (Figure 1).
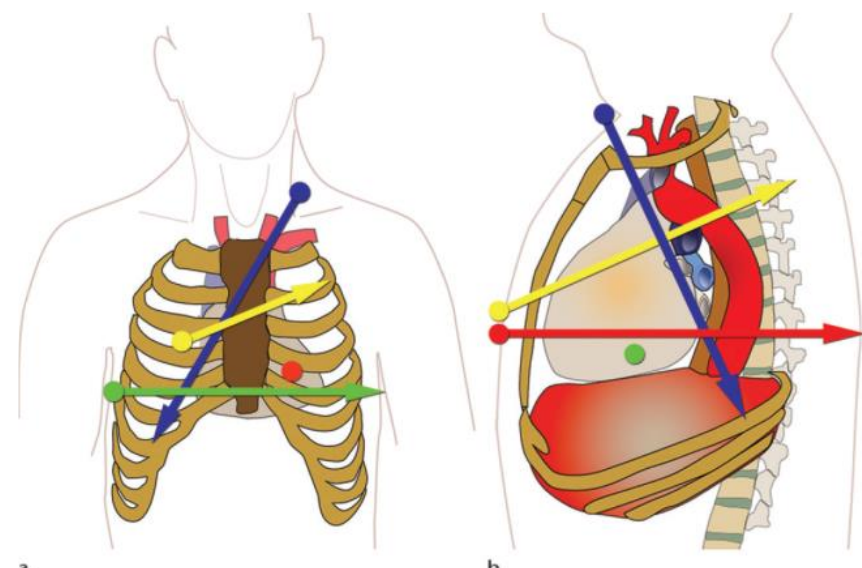

Figure 1. Drawings illustrate typical trajectories of Penetrating transmediastinal injuries (TMIs) in the frontal (a) and lateral (b) projections, including transverse (green arrow), transpulmonary (yellow arrow), and anteroposterior parasternal (red arrow) trajectories and a descending trajectory from the root of the neck (blue arrow). Colored circles $=$ corresponding skin entry sites [1]

This injury is characterized by entry and exit wounds on both sides of the thorax. A single entry wound with an image showing a firearm projectile on the opposite side of the thoracic cavity or near the mediastinum or a penetrating wound in the central region of the thorax [8]-[10].

Given the increasing incidence of this type of trauma, mainly due to the increase in the rates of violence, a growing number for gunshot wounds in our midst with high morbidity and mortality, the discussion on this topic is of high relevance and ended up to motivate the authors to perform this literature review. 


\section{METHODS}

A search was conducted in the databases Pubmed, Scopus, and Scielo using the descriptors: thorax, penetrating wounds, injury, mediastinum, thoracotomy, hospital service emergencies. The selection was performed from the reading and analysis of the title and abstract, respectively. We excluded studies whose theme did not address or was incompatible with the purpose of this review, case reports, duplicate articles that were not included in the Portuguese,
English or Spanish languages. The selection of the studies was done separately by peers, with a third researcher, in case of divergences between the other two authors. There was also research in the gray literature, so that other essential studies were included in the case of selection. The research found 66 articles, of which seven were selected for full reading, and five different types of research published in the gray literature were added, not indexed to the databases, but used in previous studies on the subject. (Figure 2).

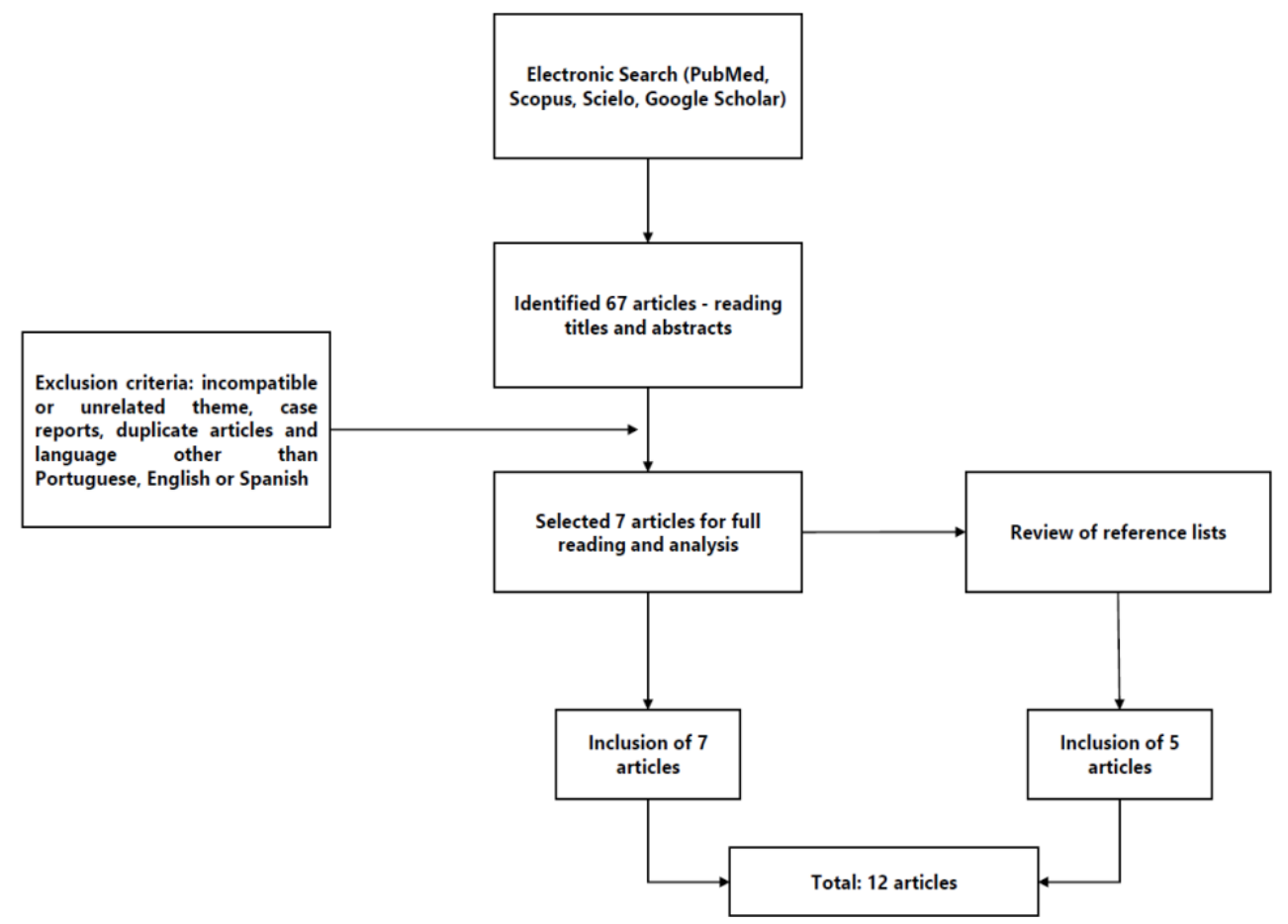

Figure 2. Flowchart for selection of included studies

\section{RESULTS AND DISCUSSION}

Mortality from this type of injury varies widely in different studies, mainly depending on the proportion of unstable patients. Many of the patients, incidentally, end up dying before being transported to the hospital due to cardiac tamponade or severe hemorrhage - Grade IV [6].

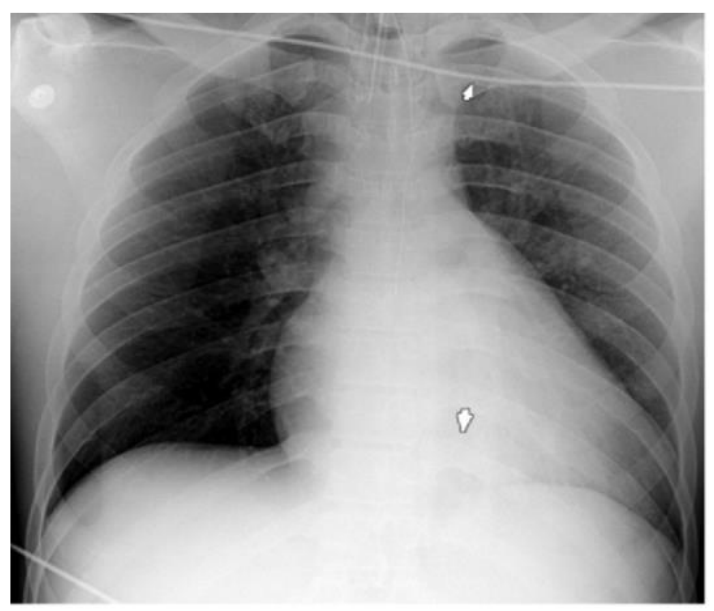

a.

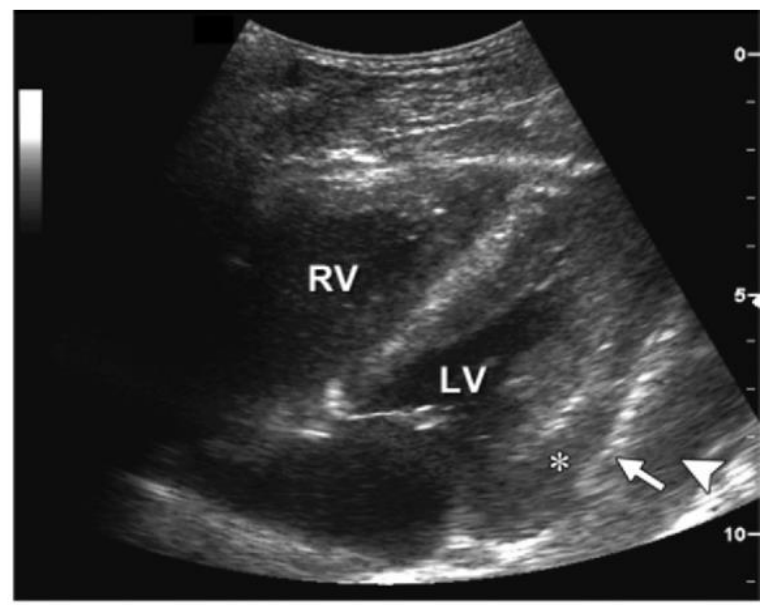

b.

Figure 3. Hemopericardium from a laceration to the anterior wall of the left ventricle in a 30-year-old man who was stabbed multiple times in the chest and abdomen. (a) Portable anteroposterior chest radiograph shows two skin entry wound markers to the left of the sternum and in the left lower chest, o verlying the heart. The cardiac contour is enlarged. (b) US image (subxiphoid view) of the heart demonstrates the parietal pericardial and pleural layers (arrow) separating a large left hemopericardium $(*)$ from a hemothorax (arrowhead) in the left pleural space. LV = left ventricle, RV = right ventricle [1]. 


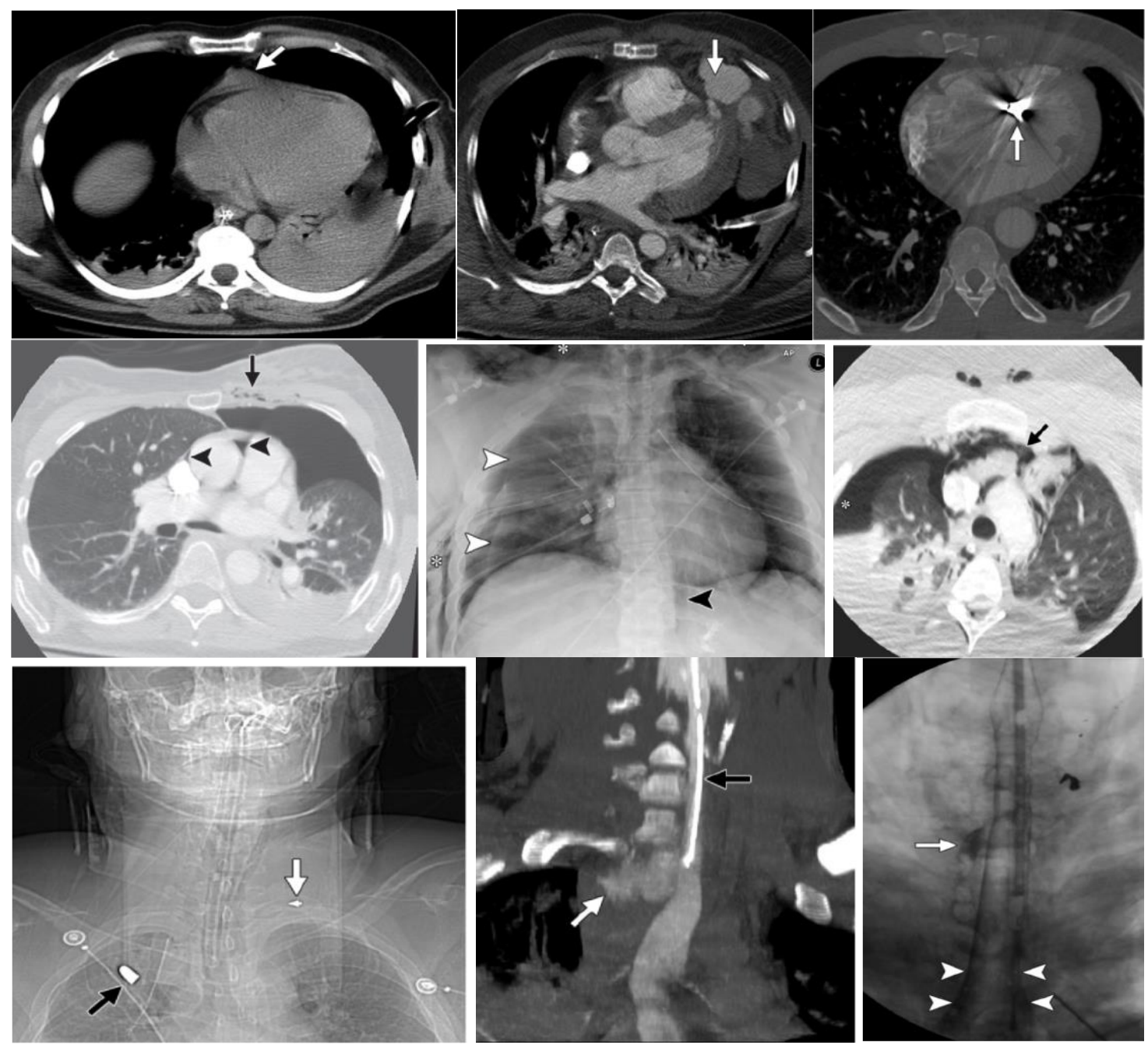

Figure 4. Several types of transmediastinal thorax lesions: cardiac, ventricular, aortic, subcutaneous emphysema, lung pneumothorax, tracheal lesion, esophageal lesion. Diagnosis by tomography of the chest, radiographs and esophagram. [1]

Bradley reported in 1966 the first series of five patients with a mortality of $20 \%{ }^{8}$. Richardson et al. prospectively studied 76 cases with an incidence of hemodynamic instability of $57 \%$ and reported a general mortality of $36 \%$ [4]. Degiannis et al. observed a general mortality of $31 \%$ in a series of 118 lesions by transmediastinal firearm, of which 57 were stable [6].

In a series of 68 patients, Renz et al. demonstrated that 32 of them had SBP $<60 \mathrm{mmHg}$ and $32 \%$ of the patients died [5]. In the study by Burack et al., a series of 207 participants, the overall mortality rate was $42 \%$ for patients with firearm projectile injuries and $7 \%$ for patients with white gun injuries, 135 of them presented hemodynamic stability and 55\% mortality in the group of hemodynamically unstable patients. In this study, mortality was also evaluated according to the trajectory of the lesion, is the transversal, caused exclusively by firearm, the highest mortality rate $(60 \%)$ [7].

The highest overall mortality rate was recorded by Okoye et al. and was mainly due to the large number of unstable patients, 116 of the 133 participants, and 97 of them were submitted to resuscitation thoracotomy [9].

When considering the mortality rate among patients undergoing resuscitation thoracotomy, the mortality rate is considerably higher. In the essay by Degiannis et al. 6 of the 7 patients submitted died [6]. Renz et al. reported 17 type of surgeries with $100 \%$ mortality [5]. On the resuscitation thoracotomy, Burack et al. reported mortality of $85 \%$ and, among the 97 patients undergoing this procedure in the study by Okoye et al., mortality was 91.8\% [7], [9].

Burack et al. observed that operative mortality was $25 \%$ for patients who survived long enough to be transferred to the operating room [10]. In other studies, operative mortality for unstable patients was $20 \%$ to $36 \%$ [7], [9].

Another important point observed by Okoye et al. was a mortality rate in patients who arrived at the emergency room without vital signs of $24.3 \%$ and no deaths in the hemodynamically stable population ${ }^{9}$. In the series of Burack et al. none of the patients in the stable group died [7].

Nagy et al. presented in a study with 50 patients, all of them hemodynamically stable, a mortality of $4 \%$, and one of the two deaths occurred from complication (pericarditis) after pericardial window [2].

A key point in taking conduct in these cases is the definition of the patient's hemodynamic status. Renz et al. was the first author to address this issue and classified the patients into three groups that took into account the value of systolic blood pressure (SBP): SBP>100 mmHg (Group I), SBP between 60-100 mmHg (group II) and SBP $<60 \mathrm{mmHg}$ (group III).

Through this assay, it was observed that patients with SBP> $100 \mathrm{mmHg}$ could be safely submitted to an additional diagnostic evaluation and that $60 \%$ of them would be treated conservatively ${ }^{5}$. In those with SBP between $60-100 \mathrm{mmHg}$ 
was the response to thoracic drainage, volume resuscitation and initial diagnostic assessment findings that determined the need for surgery, and $50 \%$ of them did not require surgical exploration in the study. However, if $\mathrm{SBP}<60 \mathrm{mmHg}$ all patients underwent emergency operation [5].

Nagy et al. considered similar parameters, defining hemodynamic stability as a pulse frequency $<100 \mathrm{bpm}$ and a systolic blood pressure $>90 \mathrm{mmHg}$ at admission or after administration in bolus fluid and chest drainage [5].

Gunn et al. have adopted similar values in the definition of hemodynamic instability: adults $<65$ years and with heart rate $>100 \mathrm{bpm}$ or systolic blood pressure (SBP) $<90 \mathrm{mmHg}$ at any time after injury [2].

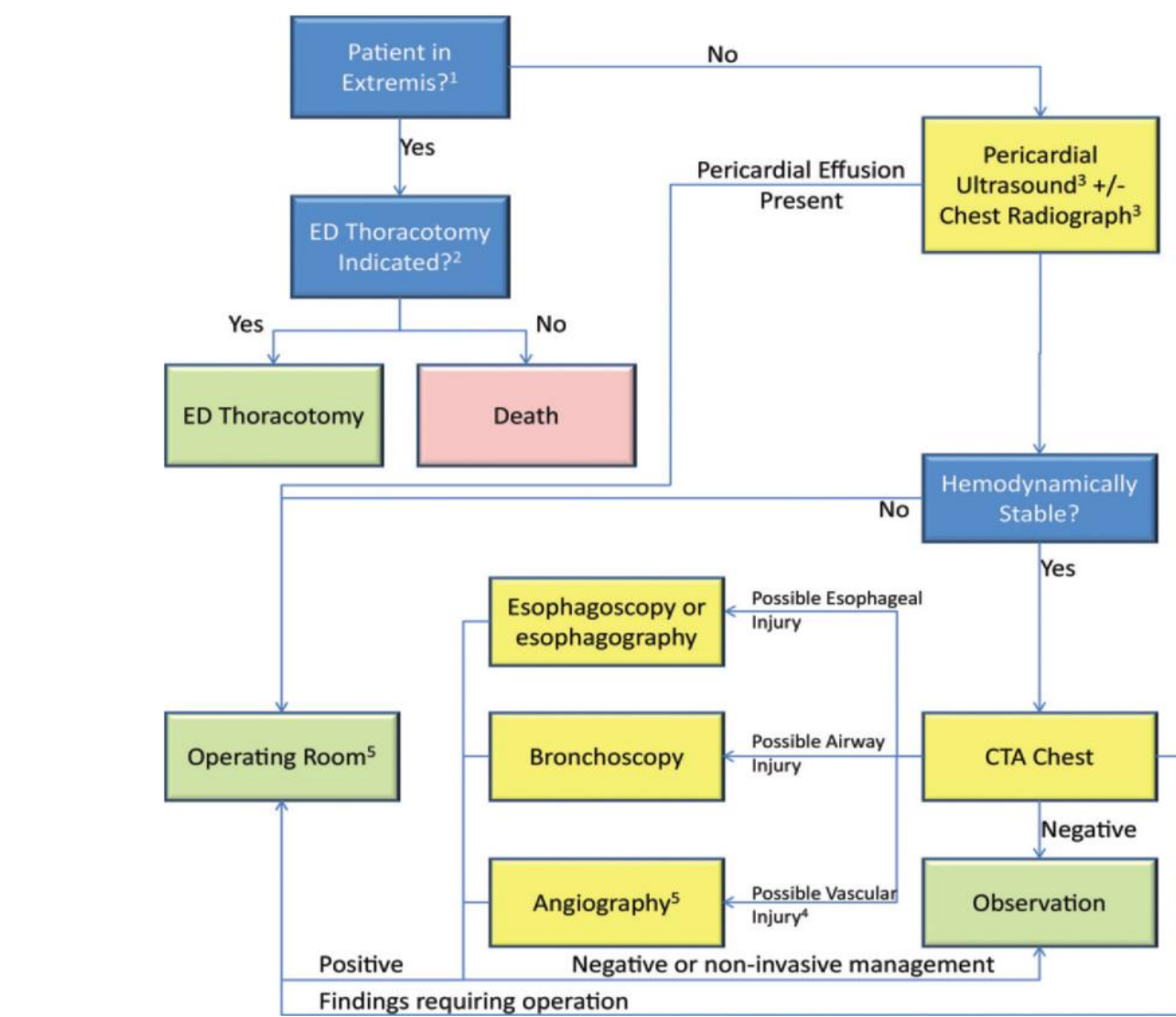

Figure 5. Chart illustrates an algorithm for the initial evaluation of patients with suspected penetrating transmediastinal trauma.[1]

Burack et al. considered hemodynamic instability when present the following situations: traumatic cardiac arrest or imminence, cardiac tamponade, persistent shock class III/IV-ATLS (2004), despite volume replacement (blood loss of $1500-2000 \mathrm{~mL}$, pulse frequency $>120 \mathrm{bpm}$, blood pressure reduction $<90 \mathrm{mmHg}$ ), output of $>1500 \mathrm{~mL}$ of blood after chest drainage, drainage $>500 \mathrm{~mL} / \mathrm{h}$ at initial time and massive hemothorax after thoracic drainage [7].

The most recent protocols/guidelines indicate that patients with recent or imminent loss of vital signs in the emergency room should undergo resuscitation thoracotomy. The other patients with hemodynamic instability should be immediately submitted to emergency thoracotomy after the realization, when possible, of chest radiography (locate fragments of firearm, identify pneumothorax and determine pathway) and/or pericardial ultrasonography at the bedside (to evaluate hemopericardium/cardiac tamponade) [3], [9], [10]. 


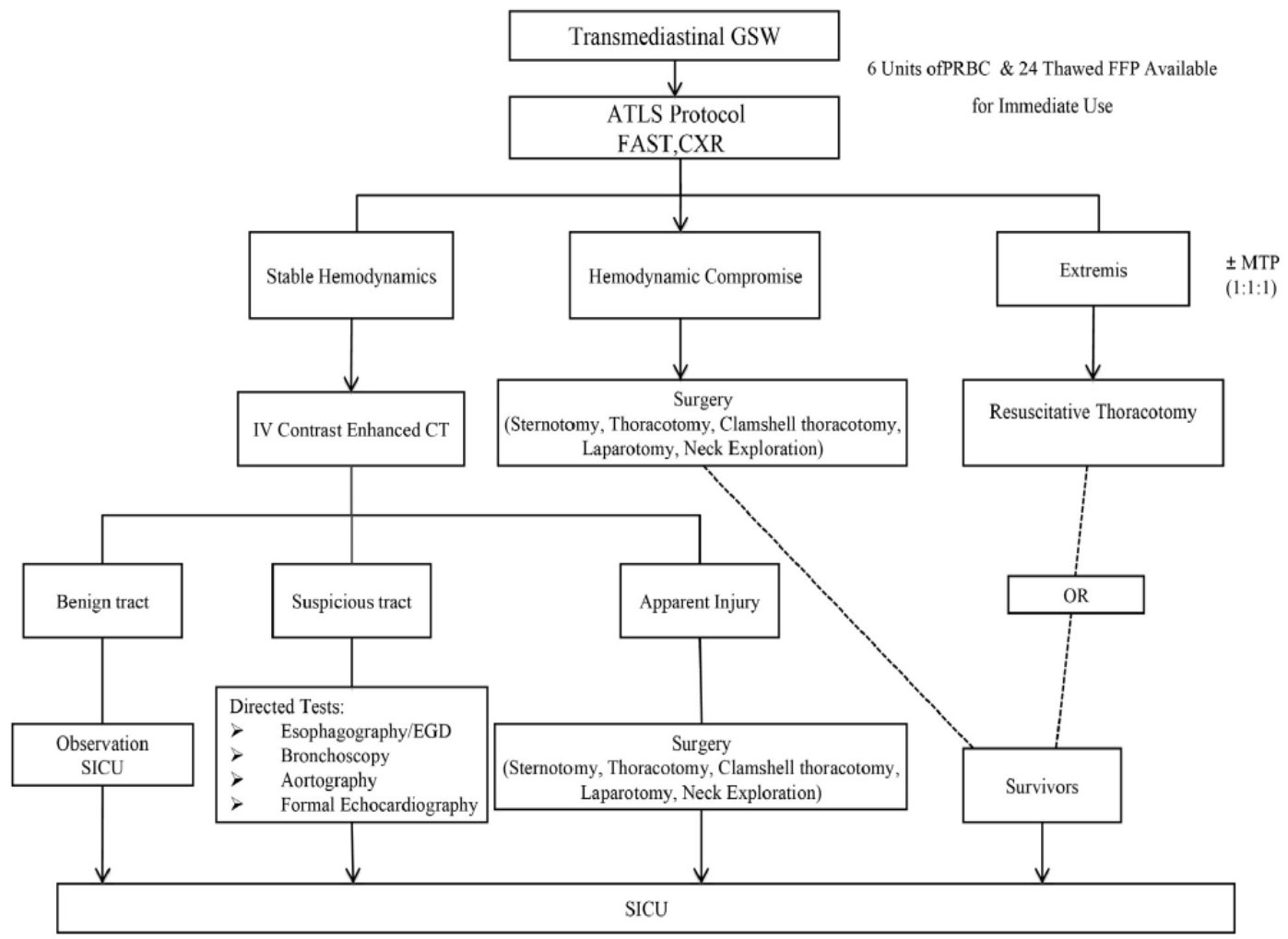

Figure 6. Algorithm for the management of transmediastinal firearm wound from LAC + USC Medical Center. In the present st udy, we used GSW: gunshot wound, FAST: focused assessment with sonography to trauma, CXR: chest X-ray, PRBC: packed red blood cells, EGF: esophago-gastro- OR: operating room, SICU: surgical intensive care unit. Source: Okoye et al. (2013) [9].

The approach of stable patients changed over time. Previously, surgical exploration was mandatory in all cases of wound by a transmediastinal firearm, if any gunshot wound that crosses an important body cavity would cause a visceral lesion, regardless of signs and symptoms [10], [11]. In the present time, all these patients were submitted to angiography, esophagography, endoscopy, bronchoscopy and pericardial window [7].

Recently, the trajectory of the projectile, in the case of gunshot wounds, has become important in the investigation of patients with a transfixant mediastinum injury [1]. In the series of cases of Nagy et al. all patients were initially evaluated by chest X-rays, being submitted to thoracostomy with drainage in water seal depending on the presence of hemo and/or pneumothorax [5].

From then on, if there is suspicion of involvement of the anterior mediastinum, the patients undergo transthoracic echocardiography or aortic angiography if the trajectory of the projectile of the firearm involves the posterior mediastinum. Patients are also submitted to esophagography and endoscopy to rule out esophageal lesions. In case of suspected tracheobronchial injury, a bronchoscopy investigation is performed [9]- [11].

Hanpeter et al. demonstrated, however, that computed tomography is reliable in assessing the trajectory of transmediastinal lesions by firearms and their potential to influence additional clinical decisions [3]. In other studies, the ability of chest CT to safely discard unnecessary examinations, considerably reduced the number of invasive procedures and the costs up to $74 \%$ [10], [11]. 

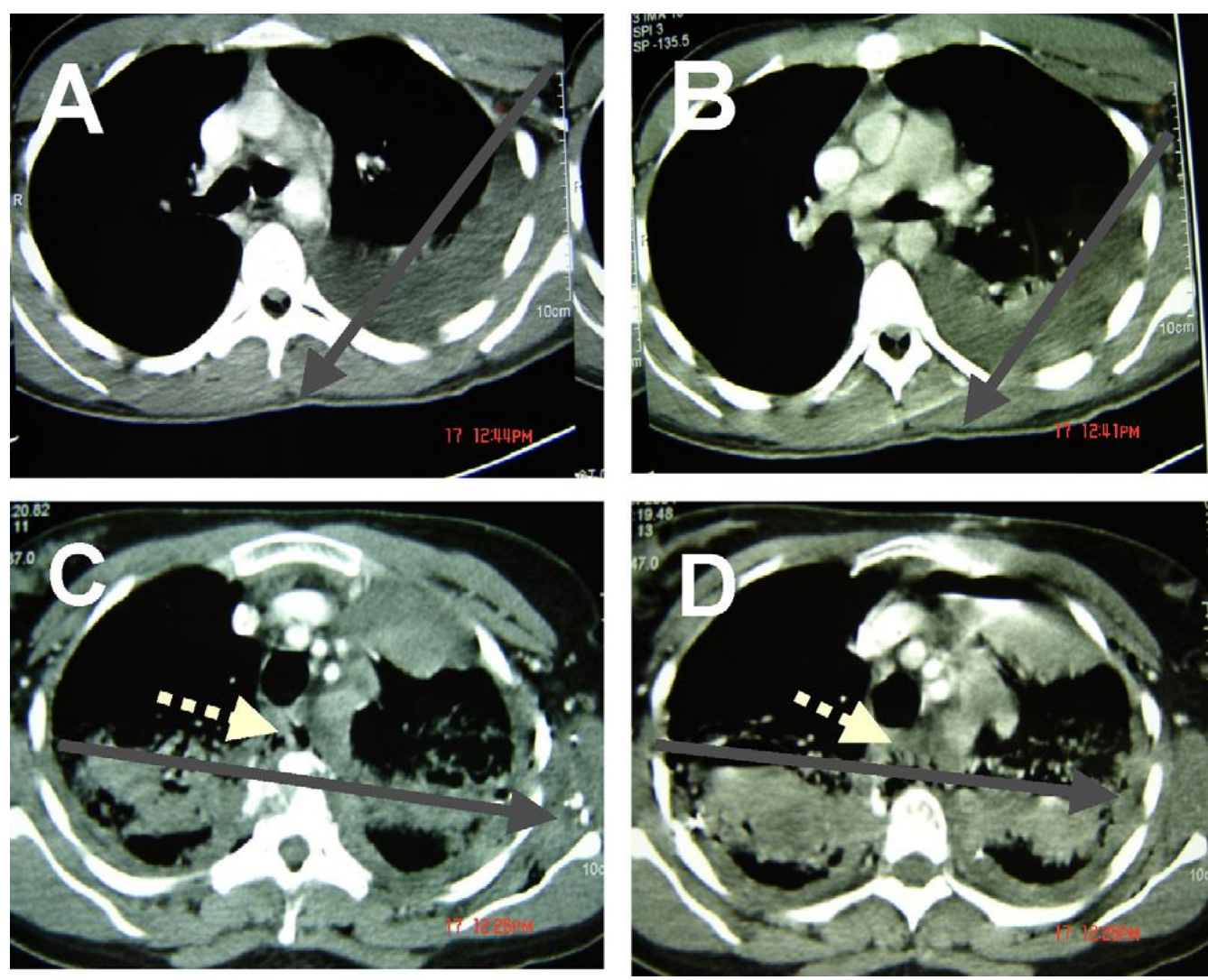

Figure 7. CTscan of thorax. (A and B) Conclusively negative CTscan (bullet tractaway from vital structures). (C and D) Inconclusive CT scan [11]

In the study conducted by Burack et al. (2007), all stable patients were initially evaluated with chest radiography, transthoracic echocardiography and CT angiography. Additional studies such as bronchoscopy, esophagography, and endoscopy were indicated only in the face of clinical suspicion, especially related to pneumomediastinum or posterior mediastinal pathway. Patients with mediastinal hematoma or trajectory suggestive of lesion to the large vessels of the bases were submitted to angiography [7].

In a more recent study, hemodynamically stable patients were evaluated with FAST (Focused Assessment with Sonography for Trauma) and computed tomography (CT) with intravenous contrast and subjected to targeted tests, including bronchoscopy, echocardiography, esophagography or catheter angiography based on clinical suspicion or radiological evaluation of lesion [8], [12], [13].

Regarding the occurrence of occult lesions in stable patients, Richardson et al. observed a $63 \%$ rate in their stable patients [7]; Degiannis et al. demonstrated this occurrence in $42 \%$; Nagy and Burack et al. obtained indices of occult lesions of $16 \%$ and $7 \%$, respectively [2], [7].

The rate of surgical exploration among stable patients ranged from $7 \%$ to $60 \%$ [5]- [7], [9], [10], [12]. This $60 \%$ index was observed in the study by Richardson et al. in which 3 of the 43 initially stable patients became unstable during the evaluation and 24 of the remaining 40 presented lesions requiring surgical intervention ${ }^{4}$. More recent studies bring rates of $7 \%$ and $17 \%$ in relation to this aspect [9], [10].

Cardiac and thoracic aortic lesions are more common in unstable patients [10]. Okoye et al. observed cardiac lesions as the most common $(70.7 \%)$, thoracic aorta $(24.1 \%)$ and pulmonary $(21.8 \%)$, paying attention to the high number of patients in extremis and with hemodynamic instability in this study [9].

Pulmonary and large vessel lesions were the most common lesions among patients surgically operated in Burack et al. [10]. Esophageal lesions may be present in $15 \%-30 \%$ of patients with posterior mediastinum lesion [7][9] and many of them do not present signs or symptoms [5], [11].

Tracheobronchial lesions are rarely found after a trasmediastinal lesion, occurring among $0-15 \%$ of stable patients [7]- [9]. These patients present suggestive clinical signs or symptoms, such as subcutaneous or mediastinal emphysema on chest $\mathrm{x}$-ray [5], [11].

The operative approach in patients with penetrating thoracic trauma depends on the hemodynamic condition and associated or suspected organic lesions. For patients in extremis, reanimation thoracotomy with aortic clamping is the only approach for rapid control of mediastinal hemorrhage. This incision can be extended through the transverse section of the sternum originating a bilateral transsternal incision (Clamshell) that offers excellent exposure of bilateral and mediastinal thoracic lesions [12]. 


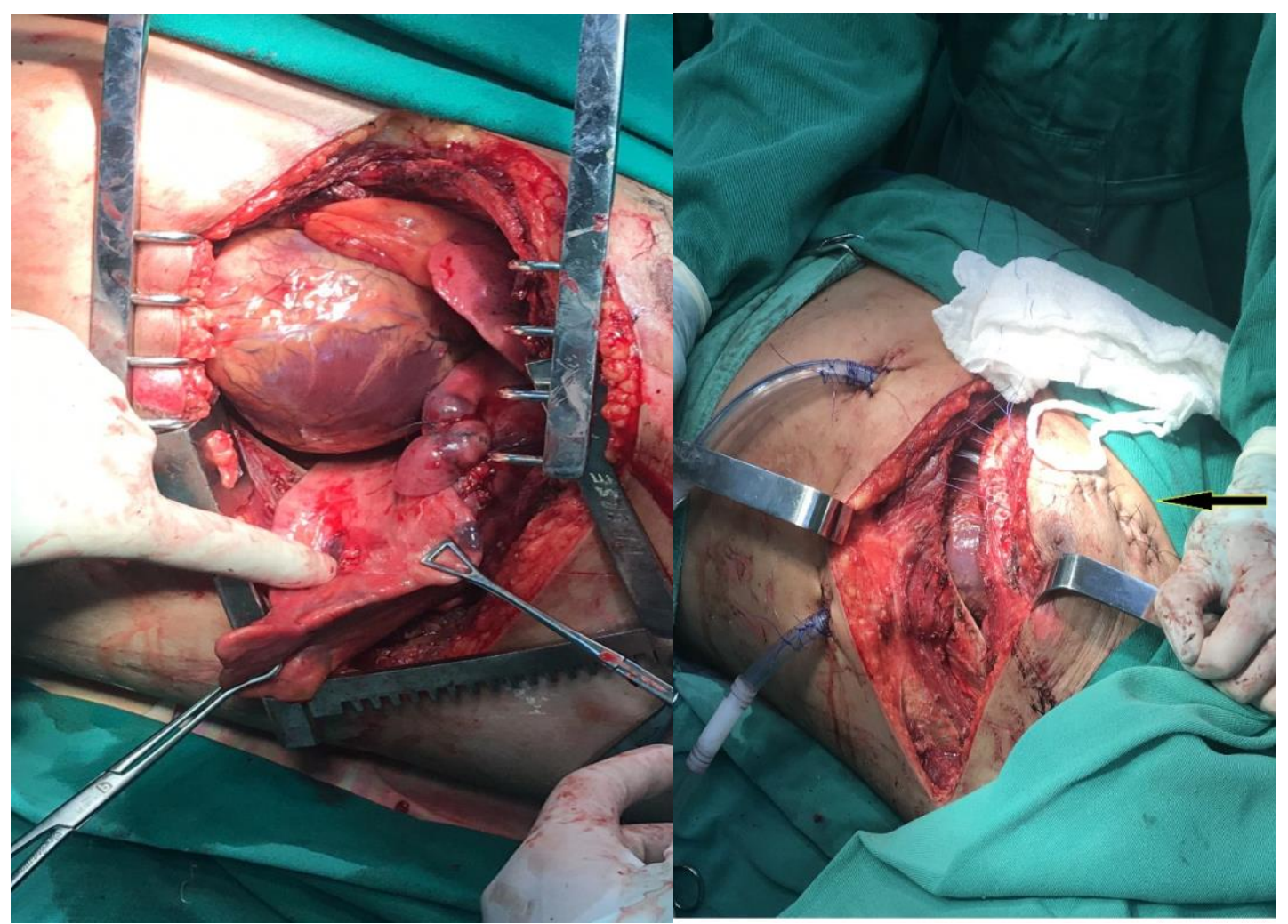

Figure 8. Resuscitation thoracotomy. Thoracic access in the fourth intercostal space. Closing of the chest after thoracotomy by knife injury in Ziedler area (arrow). Massive hemothorax after severe lung injury followed by severe hypovolemic shock, cardiorespiratory arrest, and intraoperative resuscitation after heart dislocation, defibrillation, and direct massage - Grade IV Hemorrhage (ATLS). Source: Araújo-Filho I. (2019)

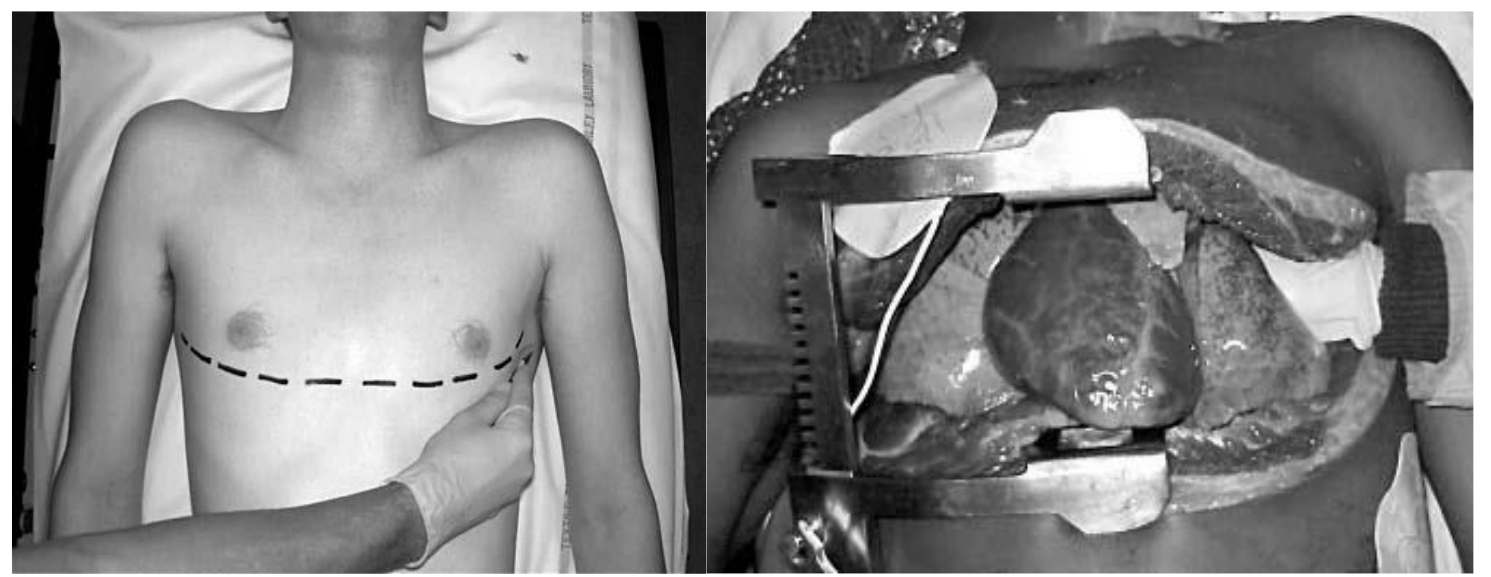

Figure 9. The Clamshell incision. In this picture the pericardium has been displaced by internal cardiac massage andlies behind the heart. Gloved hand compressing the aorta.

In stable patients with mediastinal lesions requiring surgical intervention, the incision should be adapted for optimal exposure. Most lesions can be accessed through a median sternotomy. The preferential approach, however, for lesions in the posterior mediastinum, including lesions of the descending aorta, thoracic esophagus or distal tracheobronchial structures, is lateral thoracotomy [10].

\section{CONCLUSION}

The transfixant lesions of the mediastinum are of great importance in the scenario of trauma surgery and are associated with high morbidity and mortality.

A fundamental point in the approach of these patients is the differentiation between those who are hemodynamically stable or not. In these cases, the presence or absence of hypovolemic shock and the degree of hemorrhage (ATLS) are fundamental predictors and prognostic factors.

Patients with severe hypovolemic shock/grade III/IV hemorrhages (ATLS) have a higher chance of presenting cardiac and thoracic aortic lesions and, therefore, significantly elevated mortality rates. Those arriving in extremis should be approached with resuscitation thoracotomy.

Hemodynamically unstable patients require immediate thoracotomy and can be submitted to a brief evaluation with complementary methods such as FAST. For those who are stable, they can perform chest CT scans, from which otherdirected examinations will be necessary or not.

With advances in pre-hospital care and the higher occurrence of this type of injury due to the increase of violence, the number of patients who will arrive in a situation of critical impairment of the hemodynamic state and need for resuscitation thoracotomies if will become 
more and more demanding the preparation of trauma hospitals to meet this new client profile.

In addition, large caliber firearms and high lethality require highly prepared surgical teams and trauma centers equipped to care for this type of patient, considered gravely due to the lesions they present.

Funding: none.

Conflicts of interest: none declared.

\section{REFERENCES}

[1] Gunn ML, Clark RT, Sadro CT, Linnau KF, Sandstrom CK. Current concepts in imaging evaluation of penetrating transmediastinal injury. Radiographics. 2014 Nov-Dec;34(7):1824-41.

[2] Nagy KK, Roberts RR, Smith RF, Joseph KT, An GC, Bokhari F, Barrett J. Trans-mediastinal gunshot wounds: are "stable" patients really stable? World J Surg. 2002 Oct;26(10):1247-50.

[3] Hanpeter DE, Demetriades D, Asensio JA, Berne TV, Velmahos G, Murray J. Helical computed tomographic scan in the evaluation of mediastinal gunshot wounds. J Trauma. 2000 Oct;49(4):689-94; discussion 694-5.

[4] Richardson JD, Flint LM, Snow NJ, Gray LA Jr, Trinkle JK Management of transmediastinal gunshot wounds. Surgery. 1981 Oct;90(4):671-6.

[5] Renz BM, Cava RA, Feliciano DV, Rozycki GS. Transmediastinal gunshot wounds: a prospective study. J Trauma. 2000 Mar;48(3):41621; discussion 421-2.

[6] Degiannis E, Benn CA, Leandros E, Goosen J, Boffard K, Saadia R. Transmediastinal gunshot injuries. Surgery. $2000 \mathrm{Jul} ; 128(1): 54-8$.

[7] Burack JH, Kandil E, Sawas A, O'Neill PA, Sclafani SJ, Lowery RC, Zenilman ME. Triage and outcome of patients with mediastinal penetrating trauma. Ann Thorac Surg. 2007 Feb;83(2):377-82; discussion 382.

[8] Bradley MN. Transmediastinal wounds. Am Surg. 1966 Dec;32(12):847-52.

[9] Okoye OT, Talving P, Teixeira PG, Chervonski M, Smith JA, Inaba $\mathrm{K}$, Noguchi TT, Demetriades D. Transmediastinal gunshot wounds in a mature trauma centre: changing perspectives. Injury. 2013 Sep;44(9):1198-203.

[10] Stassen NA, Lukan JK, Spain DA, Miller FB, Carrillo EH, Richardson JD, Battistella FD. Reevaluation of diagnostic procedures for transmediastinal gunshot wounds. J Trauma. 2002 Oct;53(4):6358; discussion 638.

[11] Ibirogba S, Nicol AJ, Navsaria PH. Screening helical computed tomographic scanning in haemodynamic stable patients with transmediastinal gunshot wounds. Injury. 2007 Jan;38(1):48-52.

[12] Wise D, Davies G, Coats T, Lockey D, Hyde J, Good A. Emergency thoracotomy: "how to do it". Emerg Med J. 2005 Jan;22(1):22-4. 